\title{
Propiedades puzolánicas de desechos de la industria azucarera (segunda parte)
}

\author{
Pozzolanic properties of residues of sugar \\ industries (second part)
}

J.F.MARTIRENA-HERNÁNDEZ ${ }^{1}$,S. BETANCOURT-RODRÍGUEZ ${ }^{1}$,B.MIDDENDORF²,A.RUBIO', L.MARTÍNEZFERNÁNDEZ ${ }^{1}$, I.MACHADO-LÓPEZ1 $^{1}$, R.GONZÁLEZ-LÓPEZ $^{1}$

Fecha de recepción: $31-111-99$

Fecha de aceptación: 24-VII-00
(1) Univ. central de Las Villas (CUBA)

(2) Univ. Gh Kassel(ALEMANIA)

\begin{abstract}
RESUMEN
Se presentan los resultados de estudios realizados en pastas fabricadas con un aglomerante del tipo calpuzolana, utilizando cenizas de paja de caña (CPC) y cenizas de bagazo de caña (CBC) como puzolanas. Se muestran los estudios que caracterizan la reacción de hidratación desarrollada en este tipo de aglomerante. La evaluación realizada, utilizando diferentes técnicas instrumentales, permitieron estudiar y comprender la cinética de la reacción.
\end{abstract}

\section{SUMMARY}

Results of studies conducted in lime pozzolana pastes are introduced in this paper. The pozzolana was sourced from Sugar Cane Baggasse Ash (CBC) and Sugar Cane Straw Ash (CPC). The hydration of this binder was carefully monitored by means of instrumental techniques with the aim of describing the kinetics of the reaction.

\section{ANTECEDENTES}

La información sobre el estudio de la reacción de hidratación de aglomerante cal-puzolana es escasa, y la naturaleza de la reacción es aún indefinida. En muchos casos no se recomienda la producción individual de este aglomerante, sobre todo cuando se emplean Cenizas Volantes de Carbón (PFA) y Humo de Sílice (CSF) debido a razones económicas y, a veces, por problemas de comportamiento de aplicaciones (1).

La literatura se refiere principalmente hacia aplicaciones de RHA con altas concentraciones de sílice $(2,3)$. En todos los casos se reporta la precipitación de Hidrosilicatos de Calcio (C-S-H) $(2,3,4)$. La morfología de los productos de la reacción muestra densas estructuras fibrosas que radian de los granos ricos en sílice en for- ma "procubina". Al parecer, un gel de sílice cubre los depósitos de sílice (2). Observaciones en el Microscopio Electrónico por Transmisión(TEM) muestran grupos de fibras aciculares con sección transversal hueca, que se entrecruzan entre sí. Estas morfologías son similares a las reportadas en pastas de cemento Portland hidratadas, en las que es reconocido que C-S-H son los principales productos de la reacción (3)

El mecanismo de la reacción es altamente dependiente de la concentración del ion Calcio, que se reporta con un mínimo de $2 \mathrm{mmol} / \mathrm{l}$ (3). La reacción, al parecer, no se completa en solución, y sí asistida por superficie. Este mecanismo puede ser explicado por el incremento de la solubilidad de la sílice provocada por el aumento del $\mathrm{pH}$. Cuando el pH alcanza el valor de la reacción saturada de $\mathrm{HC}$, alrededor de $25 \mathrm{Mmol} / \mathrm{l}$, la solución que rodea al 
grano de sílice reacciona con los iones de calcio para formar un precipitado gelatinoso de hidrosilicato de calcio hidratado, que envuelve al grano como una membrana semi-permeable.

La difusión del agua a través de esta membrana disuelve aún más la sílice y da lugar a la presión osmótica. Cuando la presión es alta, la membrana estalla y la solución rica en sílice se precipita hacia la solución de Calcio que la rodea. De aquí la justificación de las formas tubulares radiando de los granos de sílice. Los tubos pueden tener sus extremos cerrados o abiertos. El mecanismo de la presión osmótica puede dar una explicación elegante de las morfologías observadas en la gel de C-S-H (3).

Los estudios de Análisis Térmico Diferencial (DTA) de pastas de aglomerante cal-puzolana muestran una exoterma a temperaturas de aproximadamente $850^{\circ} \mathrm{C}$, que se ajusta perfectamente a la transición de la walastonita $(2,3,5)$, como un indicio de la presencia de C-S-H entre los productos de la reacción.

Al estudiar los restos que quedan luego de diluir PFA por 28 días en una solución rica en $\mathrm{HC}$, se detecta que todos las fases cristalinas presentes en la PFA se mantienen intactas después de estar durante este tiempo en contacto con el HC (4). Entre los principales productos de reacción están los C-S-H, que no puede, en este caso, ser identificado en los diagramas de XRD, porque su pico coincide con el de calcita. También se encuentran aluminatos de calcio Hidratados, carboaluminatos, sulfoaluminatos y trazas de etringita (4).

El tipo de curado y, mayormente, el aumento de la temperatura, se aportan como la causa principal del incremento de su resistencia mecánica, a corto y largo plazo, y del ritmo de incremento de resistencia. Esto es debido a que los aglomerantes cal-puzolana necesitan una energía de activación superior a la de los cementos Portland (6).

La resistencia mecánica es la evidencia más consistente de la ocurrencia de una reacción hidráulica cuando la cal y puzolana son molidas íntimamente y humedecidos. Diversas fuentes mencionan logros de valores significativos de resistencia a compresión en morteros. Los valores oscilan entre los $0.6 \mathrm{~N} / \mathrm{mm}^{2}$ hasta $12 \mathrm{~N} / \mathrm{mm}^{2}$ $(1,7,8,9,10,11)$. La Norma India IS 4098, que es aceptada internacionalmente para los aglomerantes calpuzolana, estipula sólo $2 \mathrm{~N} / \mathrm{mm}^{2}$ a los 7 días, y $4 \mathrm{~N} / \mathrm{mm}^{2}$ a los 28 días (11).

\section{ESTUDIO DE LA CINÉTICA DE LA REACCIÓN DE HIDRATACIÓN EN PASTAS}

Estos estudios representan el objetivo fundamental de este trabajo. Están principalmente orientados a justificar la formación de compuestos estables como resultado de la reacción de hidratación. Como se ha explicado con anterioridad, los estudios han sido realizados principalmente en pastas de consistencia normal, vertidas en moldes cilíndricos de $3 \times 5 \mathrm{~cm}$, o en prismas de $4 \times 4$ x $16 \mathrm{~cm}$. A continuación se explicarán los principales resultados obtenidos.

\section{EVOLUCIÓN DEL CONTENIDO DE HIDRÓXIDO DE CALCIO (HC) EN EL TIEMPO}

La presencia de $\mathrm{HC}$ indica claramente que la reacción de hidratación aún no ha terminado. Es lógico esperar que haya alguna cantidad de $\mathrm{HC}$ incluso a edades avanzadas (hasta un año de edad), sólo que la mayor parte del HC es consumido en el primer mes de edad. Esto sucede siempre que la muestra sea sometida a protección adecuada para impedir su carbonatación al reaccionar con el $\mathrm{CO}_{2}$ del aire.

Es posible cuantificar la presencia de $\mathrm{HC}$ por varias vías. Entre cllas se conoce de: $(12,13,2,14,9)$

Difracción de rayos $X(D R X)$. El HC tiene estructura cristalina, y es identificado como el mineral Portlandita en el difractograma. Es común asociar la intensidad del pico en el difractograma con la mayor o menor presencia del mineral en la muestra evaluada.

En la Figura 1 se identifican varios difractogramas, correspondientes a las cenizas y pastas cal-puzolana a 7 y 28 días. Es perfectamente apreciable el nuevo pico que aparece a la izquierda en el diagrama, detectable en pastas de 7 días de edad, donde aún todo el $\mathrm{HC}$ no ha reaccionado.

Evidentemente, este pico no aparece en el difractograma de las cenizas (a), que tienen un muy bajo contenido de $\mathrm{CaO}$ en su composición. Tampoco aparece en el difractograma de pastas a los 28 días de edad, debido a que la mayor parte del producto ha sido consumida, lo que indica un sustancial avance de la reacción de hidratación.

En pastas donde se ha usado CPC sólo es posible detectar $\mathrm{HC}$ en los difractogramas a la edad de 7 días. Para edades mayores no es apreciable su presencia. En pastas fabricadas sobre la base de CBC, sobre todo cuando la ceniza es obtenida directamente de las calderas, la desaparición de HC toma más tiempo, incluso a los tres meses de edad existen cantidades apreciables de $\mathrm{HC}$ sin reaccionar.

Estudios de termogravimetría. Cuando una muestra del material es sometida a tratamiento térmico, ocurren importantes cambios de fase. Para temperaturas entre los $425^{\circ} \mathrm{C}$ y $550^{\circ} \mathrm{C}$ se producen pérdidas de peso en las muestras evaluadas producto de la deshidroxilación del HC. Esta pérdida de peso produce bruscos saltos en los 


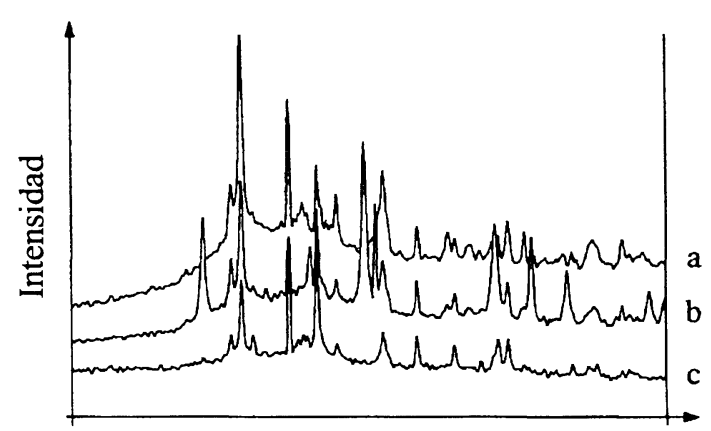

Ángulo (20)

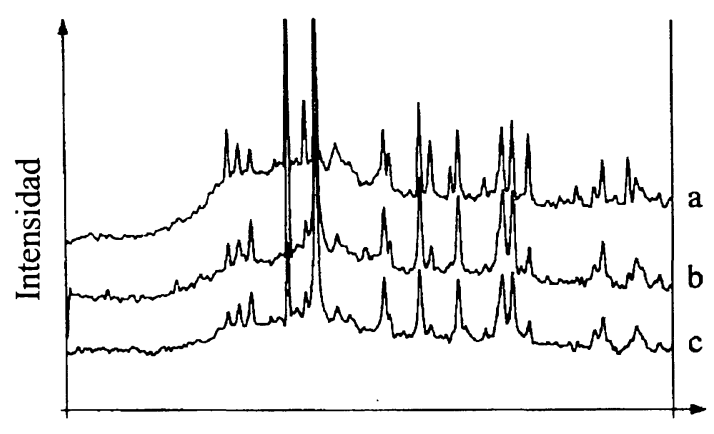

Ángulo (20)
Figura la.- Difractogramas de XRD realizados en CPC: a) cenizas, b) pastas a 7 días, c) pastas a 28 días.

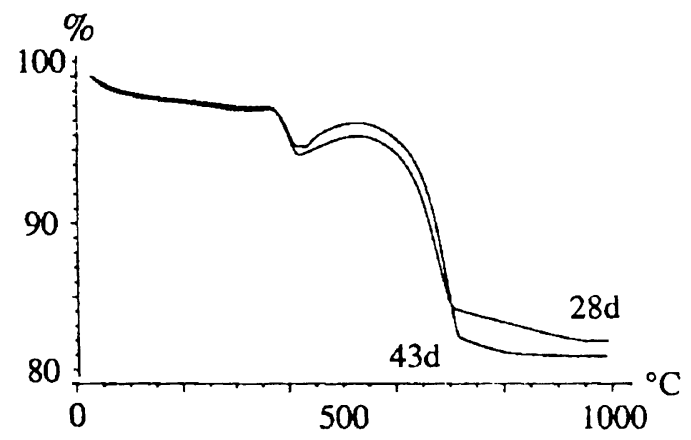

Figura 1b.- Difractogramas de XRD realizados en CBC: a) cenizas, b) pastas a 7 días, c) pastas a 28 días.

Figura 2.- Termogramas (TG) para pastas de CBC (der.) y CPC (izq.).

termogramas, y es un índice inequívoco de la presencia de $\mathrm{HC}$ en la muestra evaluada. Por medio de este estudio es posible incluso medir la cantidad de $\mathrm{HC}$ presente en la muestra, según las diferencias de peso, teniendo en cuenta los resultados de la composición química $(2,12,14)$.

Otro salto brusco ocurre a temperaturas cercanas a los $700{ }^{\circ} \mathrm{C}$, probablemente producido por la liberación de $\mathrm{CO}_{2}$ en la descomposición del $\mathrm{CaCO}_{3}$ que forma parte de la composición original de las cenizas, o que fue formado por la carbonatación de las pastas.

La Figura 2 muestra difractogramas practicados a 28 y 43 días en pastas fabricadas con CPC y CBC. Puede apreciarse la diferencia de comportamiento entre ambas muestras. En las pastas fabricadas con CPC, el escalón producido por la deshidroxilación del hidróxido cálcico es apenas imperceptible, indicio de que prácticamente todo el HC ha sido consumido en la reacción.

Por el contrario, en las pastas fabricadas con $\mathrm{CBC}$, el escalón es perfectamente perceptible, y no hay grandes diferencias entre 28 y 43 días de edad, lo que sugiere que la reacción ocurre de una forma lenta,y que aún queda una cantidad significativa de $\mathrm{HC}$ por reaccionar. Como ya se había explicado, las $\mathrm{CBC}$ son cenizas menos reactivas en comparación con las $\mathrm{CPC}$, y la reacción en estos casos es más lenta.

\section{EVOLUCIÓN DE LA ESTRUCTURA DE POROS EN EL TIEMPO}

El cambio de la estructura de poros en pastas hidratadas es asociado con la formación de productos de reacción. La precipitación de estos productos densifica la red intrincada, creada inicialmente por el aire ocluido, los conductos capilares y los poros de gel.

Este fenómeno produce un incremento de los poros de gel, debido, fundamentalmente, a que los nuevos productos se producen precisamente dentro de los poros capilares y los subdividen de forma progresiva hasta que por su tamaño se convierten, a su vez, en poros de gel. O sea, un indicio de madurez de la reacción lo es el aumento de la proporción de poros de gel (radio $<30 \mathrm{~nm}$ ) a costa de la disminución de los poros capilares $(30 \mathrm{~nm}$ $>$ radio $\left.<10^{4}\right) \mathrm{y}$, en menor medida, los poros por aire ocluido (radio $\left.>10^{4} \mathrm{~nm}\right)(2-4,14)$.

La Tabla 1 presenta los resultados de un estudio comparativo de la estructura de poros de diferentes grupos de pastas, todas fabricadas con CPC y CBC. Se introduce un nuevo grupo de pastas, consistente en Cemento Portland Ordinario (CPO), mezclado con $\mathrm{CPC} / \mathrm{CBC}$.

Para el estudio se prepararon tres grupos de control. Los dos primeros son pastas fabricadas con RHA en forma de 
TABLA 1

Estructura de poros en pastas hidratadas

\begin{tabular}{|c|l|c|c|c|c|}
\hline $\mathrm{Nu}$ & Muestra & Aire oclui. \%) & Capilares(\%) & Gel (\%) & Por Tot (\%) \\
\hline 1 & Cal-puzolana CBC & 4.06 & 37.99 & 3.83 & 45.89 \\
\hline & Cal-puzolana CPC & 3.89 & 28.52 & 19.24 & 51.67 \\
\hline & Cal-puzolana RHA & 3.44 & 17.09 & 25.24 & 45.78 \\
\hline 2 & CPO (70\%)+ CBC (30\%) & 4.63 & 15.47 & 19.79 & 39.89 \\
\hline & CPO (70\%)+ CPC (30\%) & 4.58 & 17.2 & 20.62 & 42.4 \\
\hline & 3.5 & 19.7 & 10.6 & 34.15 \\
\hline $\begin{array}{l}\text { CPO (70\%)+RHA(30\%) } \\
\text { (control) }\end{array}$ & 3.6 & 24.2 & 10.4 & 38.17 \\
\hline 3 & Grupo control (100\% CPO) & & &
\end{tabular}

cal puzolana y CPO mezclado. El tercer grupo incluye pastas fabricadas con CPO puro. El objetivo es comparar diferentes estados de madurez de la reacción, a partir de la evolución de la estructura de poros y la porosidad total.

Como es de esperar, la máxima porosidad total está en las pastas de cal-puzolana. La distribución de poros de las pastas cal-CPC es similar a la de la serie de control (calRHA). Sin embargo, las pastas de cal-CBC tienen un comportamiento diferente. El contenido de poros de gel es insignificante, mientras que el porciento de poros capilares es significativamente mayor que en el resto de las muestras.

La comparación de la estructura de poros de las pastas de cal-puzolana con las de cemento mezclado $(\mathrm{CPO} /$ puzolana) permite inferir diferencias en el grado de madurez de la reacción de hidratación en cada tipo de material. Sin embargo, la distribución de poros es muy parecida en ambas muestras.

\section{IDENTIFICACIÓN DE NUEVOS PRODUCTOS DE LA REACCIÓN}

El principal producto de la reacción entre $\mathrm{HC}$ y una puzolana debe ser C-S-H, que se produce cuando la sílice amorfa reacciona con el HC. Sin embargo, no hay mucha información disponible sobre la naturaleza de esta reacción (2-4).

Los difractogramas para pastas de CPC/CBC confirman la aparición de un nuevo pico, que corresponde muy bien con el de C-S-H. Este pico aparece durante los primeros 7 días después de hidratar las pastas. $\mathrm{Al}$ principio se produjo cierta confusión por la cercanía del pico de la calcita (presente en las cenizas), pero, al tiempo que la reacción evolucionó, la intensidad de los picos principales y secundarios de C-S-H se incrementó significativamente, lo que permitió eliminar las dudas (ver Figura 1).

La observación de las muestras de pastas de cal-CPC/ $\mathrm{CBC}$ en el Microscopio Electrónico de Barrido (SEM), a los 28 días de edad, permitió confirmar, de forma definitiva, la presencia de fases de C-S-H en forma de agujas fibrosas tronco-cónicas, que radian desde los granos de la puzolana. El grupo de control (RHA) mostró estructuras similares (2-3).

Aunque las agujas de C-S-H fueron detectadas en ambas pastas, la mayor cantidad de ellas estuvo en las pastas de cal-CPC. La Figura 3 muestra las fases de C-S-H detectadas en las pastas de cal-CPC, tienen morfologías muy similares a las detectadas en pastas de $\mathrm{CPO}$ de 28 días de edad, correspondientes a fases de C-S-H del tipo II (14).

De forma adicional se hicieron estudios usando técnicas de Energía de Dispersión de Rayos X (EDX) para identificar los principales componentes presentes en los supuestos nuevos productos de la reacción y compararlos con sus similares en la muestra de CPO evaluada. Los resultados fueron altamente positivos, ya que, en ambos casos, fueron identificados los mismos elementos $(\mathrm{Ca}$, $\mathrm{Si}$ ). Esto representa una prueba adicional para confirmar la presencia de estructuras de Hidrosilicatos de Calcio en las pastas de cal-puzolana.

\section{PROPIEDADES MECÁNICAS EN PASTAS}

El cambio en la microestructura de un material afecta necesariamente sus macropropiedades, entre ellas las propiedades mecánicas, su resistencia a compresión y flexo-tracción. Ambas son indicadores confiables para evaluar la evolución de la reacción, ya que la formación de nuevos productos hace que la estructura del material sea más densa, y por ende, más resistente.

Las pastas, fabricadas sobre la base de cal/CPC/CBC fueron moldeadas en prismas de $4 \times 4 \times 16 \mathrm{~cm}$ y sometidas a cargas de compresión a 7 y 28 días. Para su curado fueron conservadas bajo condiciones especiales, evitando la carbonatación.

La Tabla 2 muestra los resultados de estos ensayos. Se puede observar que las mejores propiedades mecánicas corresponden a las pastas fabricadas con $\mathrm{CPC}$, como era de esperar luego de interpretar los estudios anteriores. A los 
TABLA2

Resultado de las pruebas de resistencia a compresión realizadas

\begin{tabular}{|l|c|c|}
\hline Muestra & Edad & Resistencia a compresión (MPa) \\
\hline Cal-puzolana CBC & 7 & 2.7 \\
\hline Cal-puzolana CBC & 28 & 7.7 \\
\hline Cal-puzolana CPC & 7 & 7.2 \\
\hline Cal-puzolana CPC & 28 & 13.7 \\
\hline
\end{tabular}
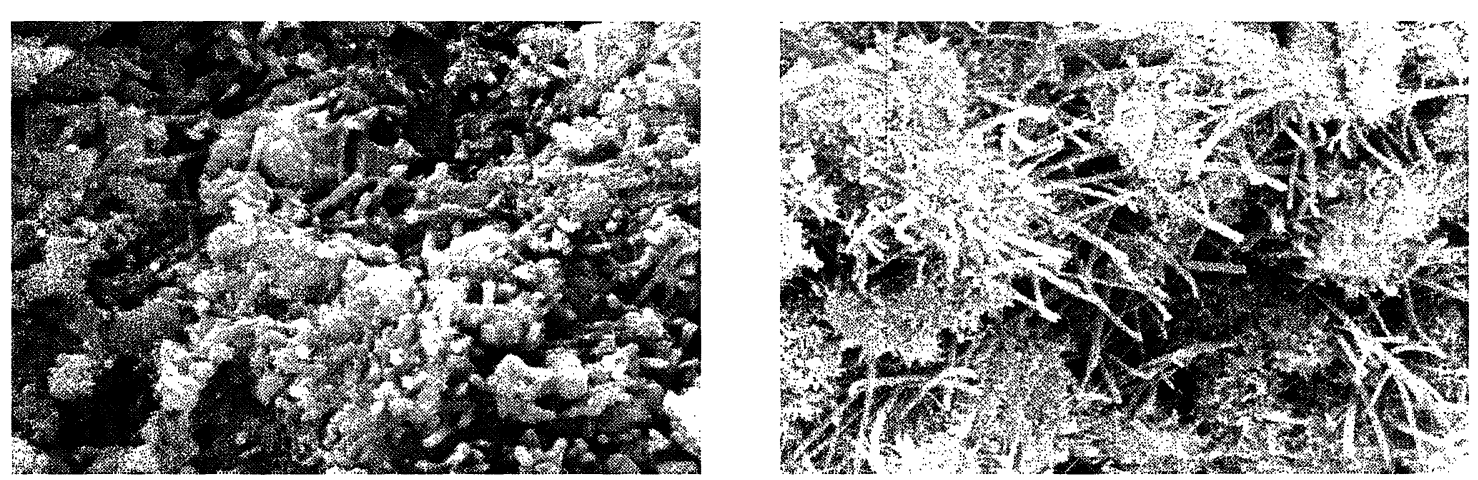

Figura 3.- Fotos de SEM de pastas CPC (izq.) y CPO (der.).

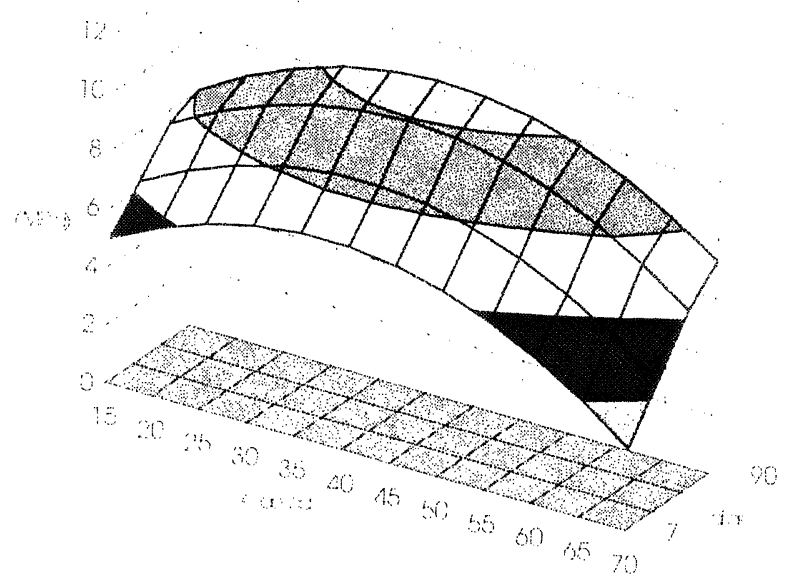

Figura 4.- Modelo probabilístico de ajuste de resistencia a compresión vs \% cal.

28 días estas pastas duplican la resistencia a compresión alcanzada a los 7 días, lo que demuestra que, a esta edad, la reacción ha alcanzado suficiente grado de madurez. Las pastas cal/CBC tienen un peor comportamiento, explicado por la baja reactividad de las cenizas utilizadas.

Adicionalmente se han hecho varios estudios para evaluar la resistencia en morteros fabricados con aglomerante cal-puzolana. De hecho, una de las formas de determinar las proporciones de cal y puzolana es hacer pruebas experimentales de las propiedades mecánicas de morteros fabricados con varias combinaciones de cal y puzolana, $\mathrm{y}$, sobre la base de modelos estadísticos poder determinar la proporción óptima.
La Figura 4 muestra un ejemplo de la evaluación de la influencia de la proporción cal-puzolana en las propiedades mecánicas de la mezcla. Para el procesamiento de los resultados se emplearon modelos probabilísticos de multivariable, que brindaron excelentes resultados. Se puede observar que cuando se emplea entre un $25-35 \%$ de cal (o sea, un 65-75\% de puzolana, en peso) se logran los mejores valores de resistencia mecánica. Estos resultados son confirmados por la determinación de las relaciones molares (9).

\section{CONCLUSIONES}

Las principales conclusiones de este trabajo son: 
Se ha podido confirmar la formación de compuestos estables durante la reacción puzolánica cuando se emplean desechos de la industria azucarera como fuente de puzolana. Los principales compuestos formados son los hidrosilicatos de calcio de forma compleja. Esto ha sido demostrado de forma conclusiva por:

a) Estudio de la evolución en el consumo del $\mathrm{HC}$ en la reacción. En pastas, usando CPC, este proceso ocurre, en su mayor parte, en los primeros 28 días. En pastas con CBC el proceso es un poco más lento, por la baja reactividad de las cenizas.

b) Estudio de la evolución de la estructura de poros en pastas hidratadas. La estructura de poros a los 28 días en pastas de cal-puzolana es similar a la de pastas de CPO, sólo que con un grado menor de madurez. El incremento de formación de poros de gel es un signo inequívoco de la precipitación de nuevos productos de reacción, que ocupan los espacios libres entre los granos.

c) Observación de los productos de la reacción. Han sido detectadas fases de C-S-H tanto en los difractogramas (XRD), como observadas directamente en el SEM. Las fases de C-S-H se presentan en forma de agujas fibrosas, que radian de los granos ricos en sílice. Este comportamiento se ajusta perfectamente al modclo de la presión osmótica, aceptado para esta reacción en la literatura consultada.

d) Pruebas de resistencia mecánica. Las pastas moldeadas en forma de prismas y sometidas a ensayos de compresión, mucstran un excelente comportamiento mecánico, lo que permite confirmar todos los resultados de los estudios anteriores. Las pastas fabricadas con cal/ CPC presentan las mejores propiedades mecánicas.

\section{BIBLIOGRAFÍA}

(1) Malhorta S.K.: Cementitious Binders from Agro-Industrial By-Products. BASIN NEWS. U.K. 1993.

(2) James J., Subba Rao M.: Reaction Product of Lime and Silica from the Rice Husk Ash., Cement and Concrete Research, Vol. 16, pp 67$73,1986$.

(3) James J., Subba Rao M.: Reactivity of rice Husk Ash., Cement and Concrete Research, Vol. 16, pp 296-302, 1986.

(4) Luxan M.P., Sanchez de Rojas M.I. and Frias M.:Investigation of the Fly Ash-Calcium Hydroxide Reactions., Cement and Concrete Research, Vol. 19, pp 69-80, 1989.

(5) Khangaonkar P.R., Azmi Rahmat : Kinetic Study of the Hydrothermal reaction Between Lime and Rice Husk Ash-Silica., Cement and Concrete Research, Vol. 22, pp 577-588, 1992.

(6) Caijun S., Day R.L.,: Acceleration of the Strength Gain of Lime Pozzolana Cements by Thermal Activation., Cement and Concrete Research, Vol. 23,pp 824-832, 1993.

(7) Martirena J.F.:The Development of Pozzolanic Cement in Cuba., Journal of Appropriate Technology, Vol. 21, No. 2 , September 1994, Intermediate Technology Publications, U.K.

(8) Mehta K.: The Chemistry and Technology of Rice Husk Ash Cements., Proceeding of the Joint Work Shop organized by UNIDO in Pakistan, 22-26 January 1979.

(9) Rabilero A.: Una contribucion al estudio del aglomerante cal-puzolana. Reporte al IX Forum de Ciencia y Tecnica (in spanish). Havana.Cuba. 1993.

(10) Smith R.G.: Rice Husk Ash Cement., Paper presented at the First International Seminar on Lime and Other Alternative Cements, Stoneleigh, Warwickshire, U.K., 9-11 Dec. 1991.

(11) Mehrotra and Irshad Masood: Pozzolanic Behaviour of Bagasse Ash. Building Research and Information, Vol. 20, Number 5,pp 299304, 1992.

(12) Arjunan P., Kumar A.: Rapid techniques for Determination of Free Lime and Free Magnesia in Cement Clinker and Portlandite in Hydrates., Cement and Concrete Research, Vol. 24, pp 343-352, 1994.

(13)Day R.L. and Caijun S.: Effect of Initial Water Curing on the Hydration of Cements Containing Natural Pozzolana., Cement and Concrete Research, Vol. 24,pp 463-472, 1994.

(14) van Roode M., Douglas E., Hemmings R.T.: X-Ray Diffraction Measurement of Glass Content in Fly Ashes and Slags., Cement and Concrete Research, Vol. 17, pp 183-197, 1987. 